\title{
LATE HOLOCENE RAISED SHORE PLATFORMS IN SOUTHWESTERN JAMAICA
}

Kevin Ray Evans, Missouri State University Toby J. Dogwiler, Missouri State University Douglas J. Faulkner University of Wisconsin-Eau Claire Peter M. Jacobs, University of Wisconsin-Whitewater Brett-M.Kenning, Missouri State University Scott A. Lecce, East Carolina University Robert T. Pavlowsky, Missouri State University and Ozarks Environmental and Water Resource Institute
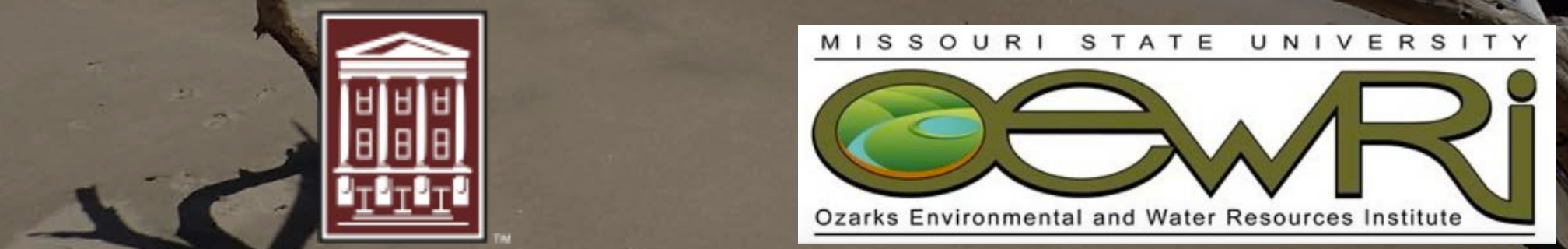

\section{Missouristate.}




\section{KEY POINTS}

Hundreds of raised shore platforms along SW Jamaica

- Typical tidal range around $20-30 \mathrm{~cm}$

- Shore platforms encrusted with sub-fossil serpulid worms

Ltving serpulids at sea level and below

- ${ }^{14} \mathrm{C}$ age date $1184 \in 940 \mathrm{cal}$ BP $[956 \pm 122 \mathrm{CE}](95.4 \%$ C])

- Wave-cut notches below shore platforms

- Abrupt(?) uplift of about $1 \mathrm{~m}$

- If single event, potentially a M6-M7 event

- Coastal features are a disappearing resource... at risk from erosion and climate change 


\section{TECTONIC SETTING}

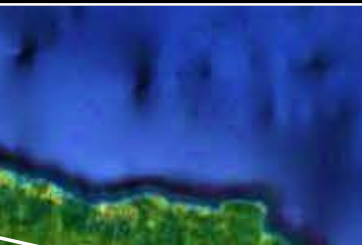

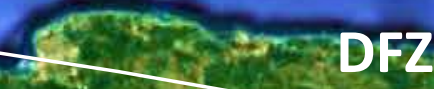

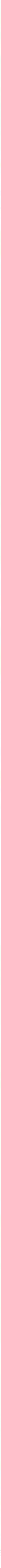




\section{GEOLOGIC SETTING}

- 140 million years in age

- Cretaceous volcanics

- K-Pg Rift zones

- Yellow Ls Group (mid-Eo.)

- White Ls Group (mid-Eo. to mid-Mio.)

- Heavily faulted and uplifted

- Coastal Group (mid-Mio. to Pleist.)

- Seismically active

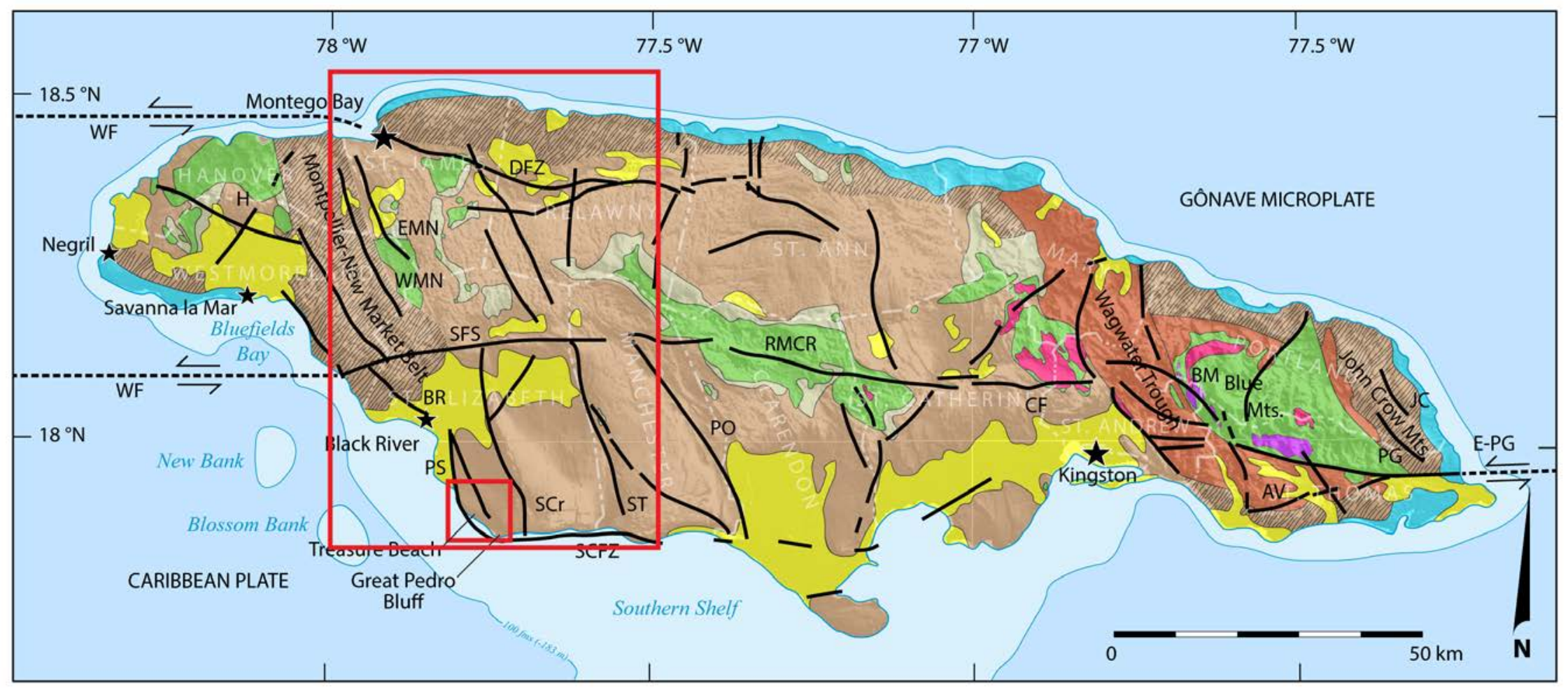

EXPLANATION

Alluvium (Quaternary sediments and morass)

Coastal Group (mid-Miocene to Pleistocene)

White Limestone, shallow/deep water facies (mid-Eocene to mid-Miocene)

Yellow Limestone (Paleocene)

Wagwater and John Crow rift deposits

Cretaceous sedimentary rocks (with granitoids and volcanics)

$\leftrightharpoons$ Faults, arrows indicate relative movement
MAJOR FAULTS AND FAULT ZONES

AV Aeolus Valley

BM Blue Mountain

BR Black River

CF Cavaliers Fault

DFZ Duanvale Fault Zone

EMN Eastern Montpellier-New Market

E-PG Enriquillo-Plantain Garden

JC John Crow

PG Plantain Garden

$\star \quad$ Key urban areas
H Hanover 


\section{STRUCTURAL SETTING}

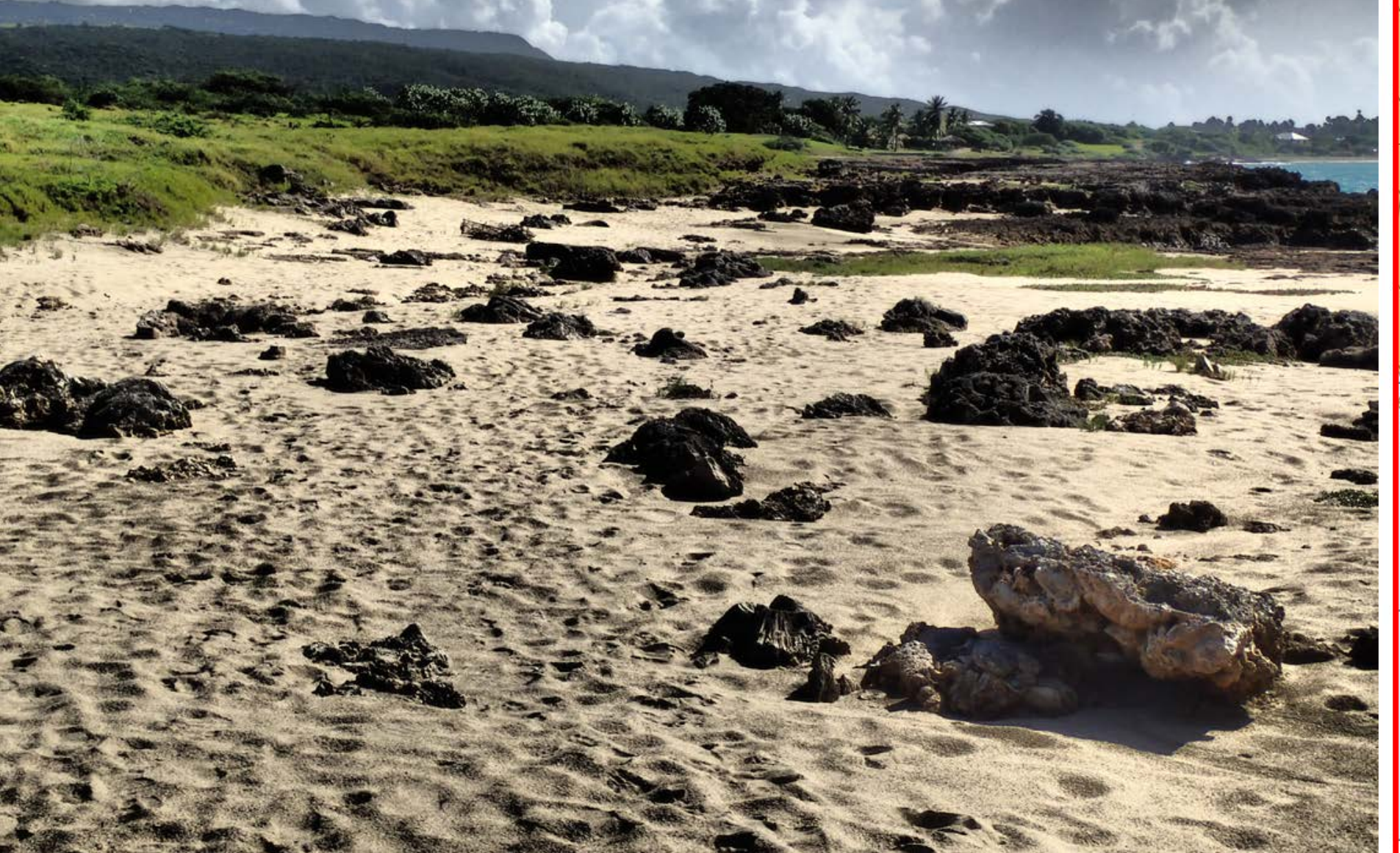

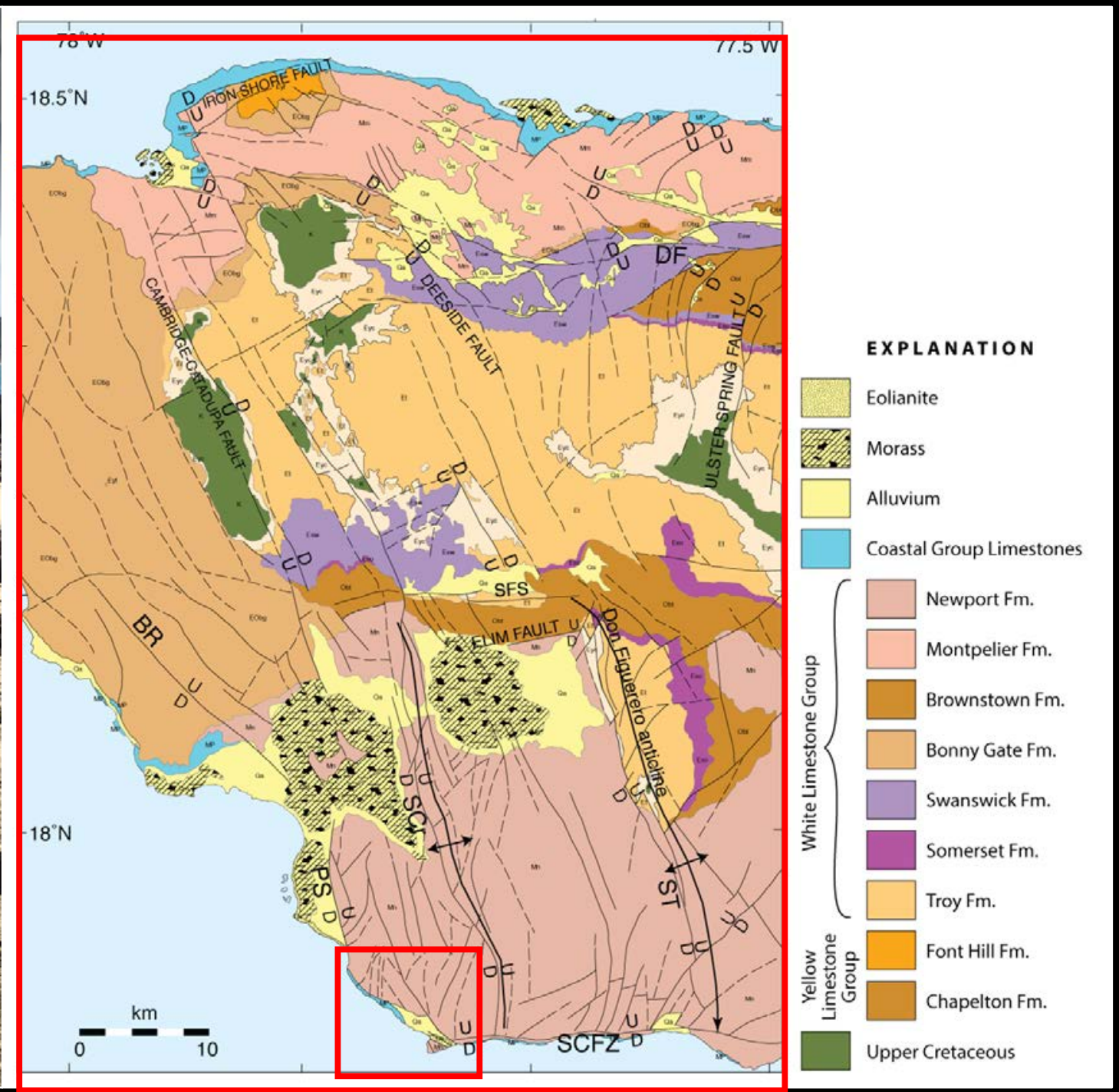

Modified from Benford et al. (2014) and Walker (1975) 


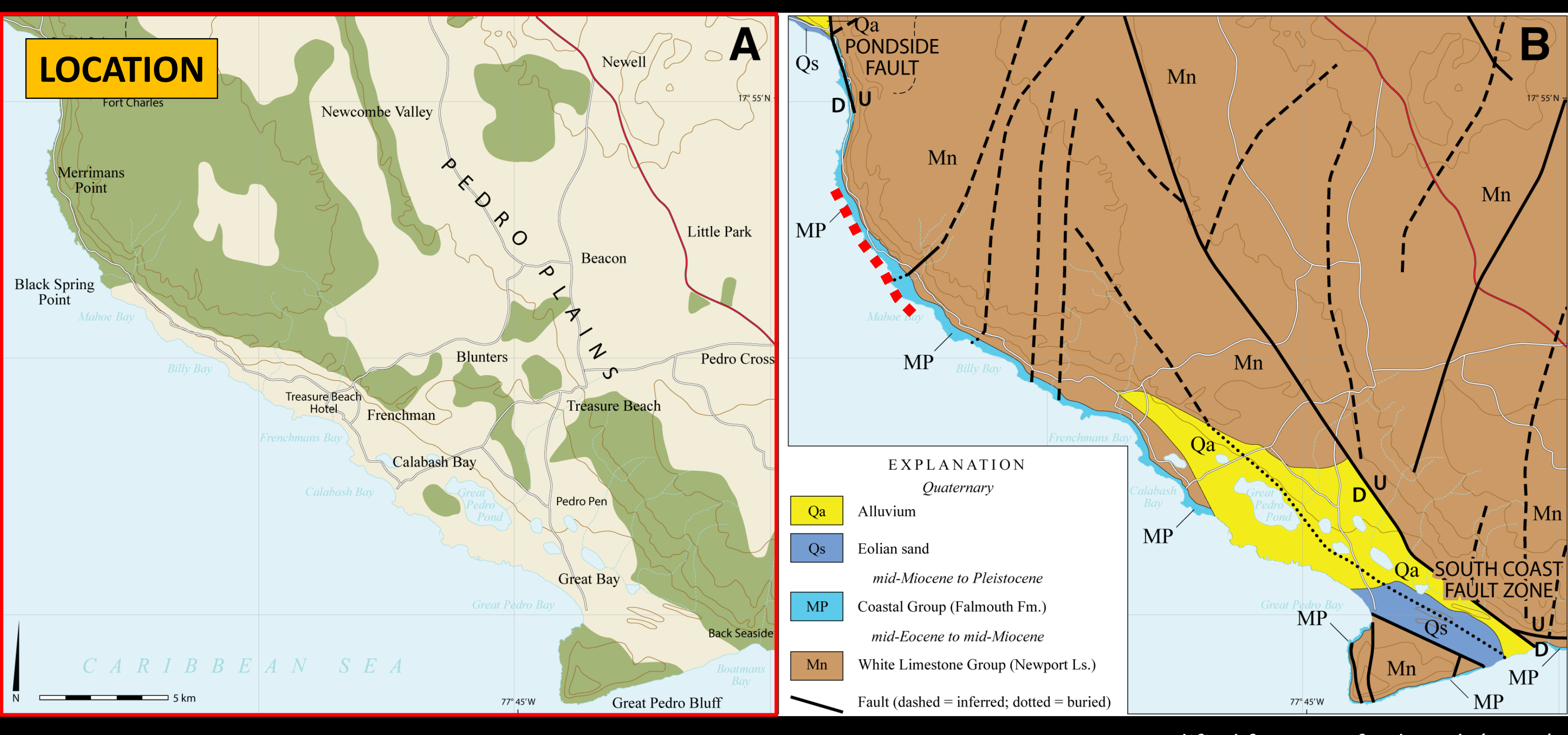




\section{DIGITAL ELEVATION MODEL}

Fort Charles Bay

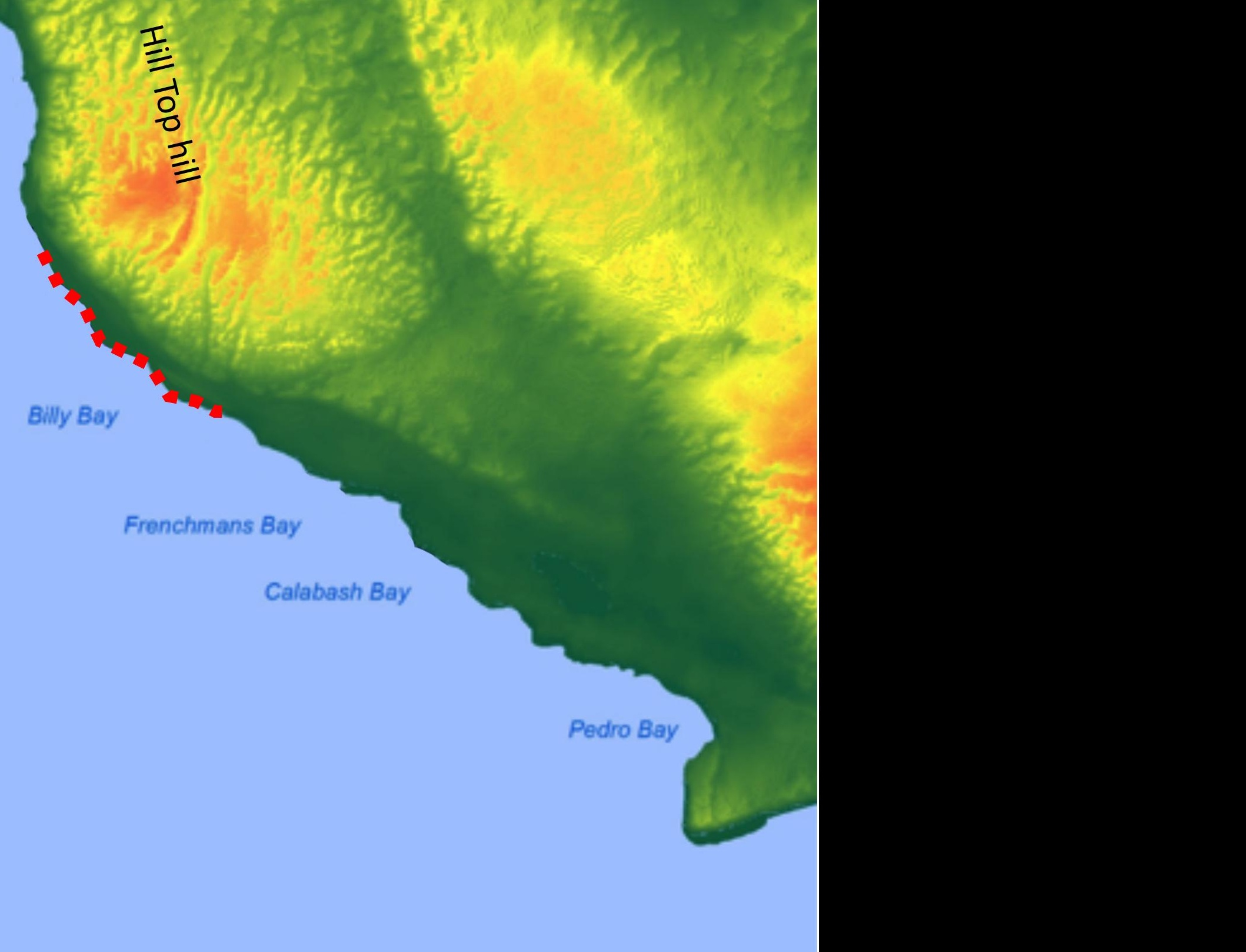




\section{OBLIQUE SATELLITE VIEW}
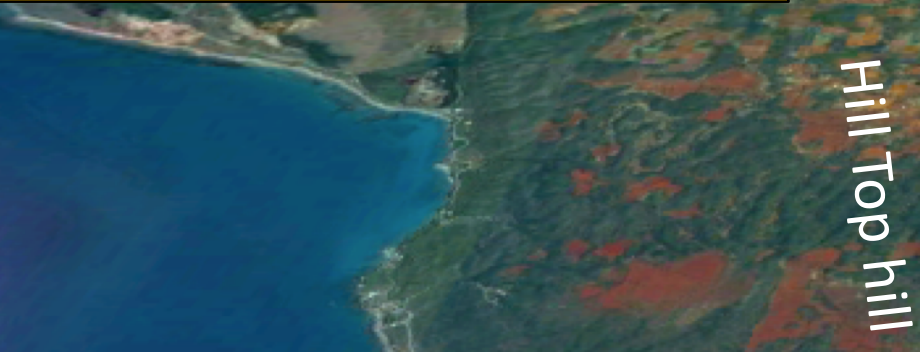

产

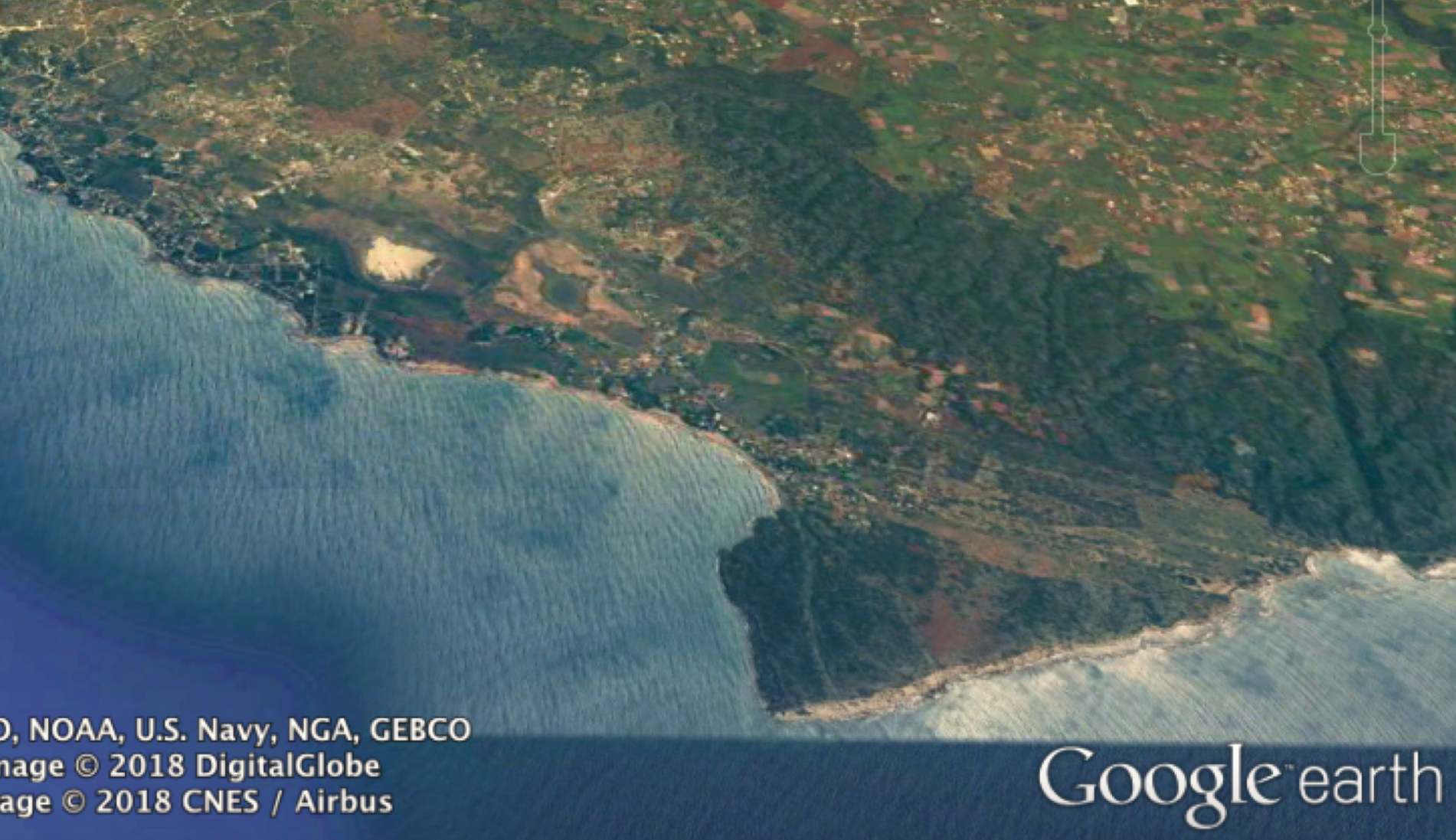




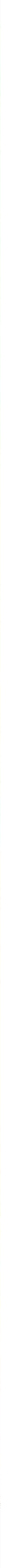




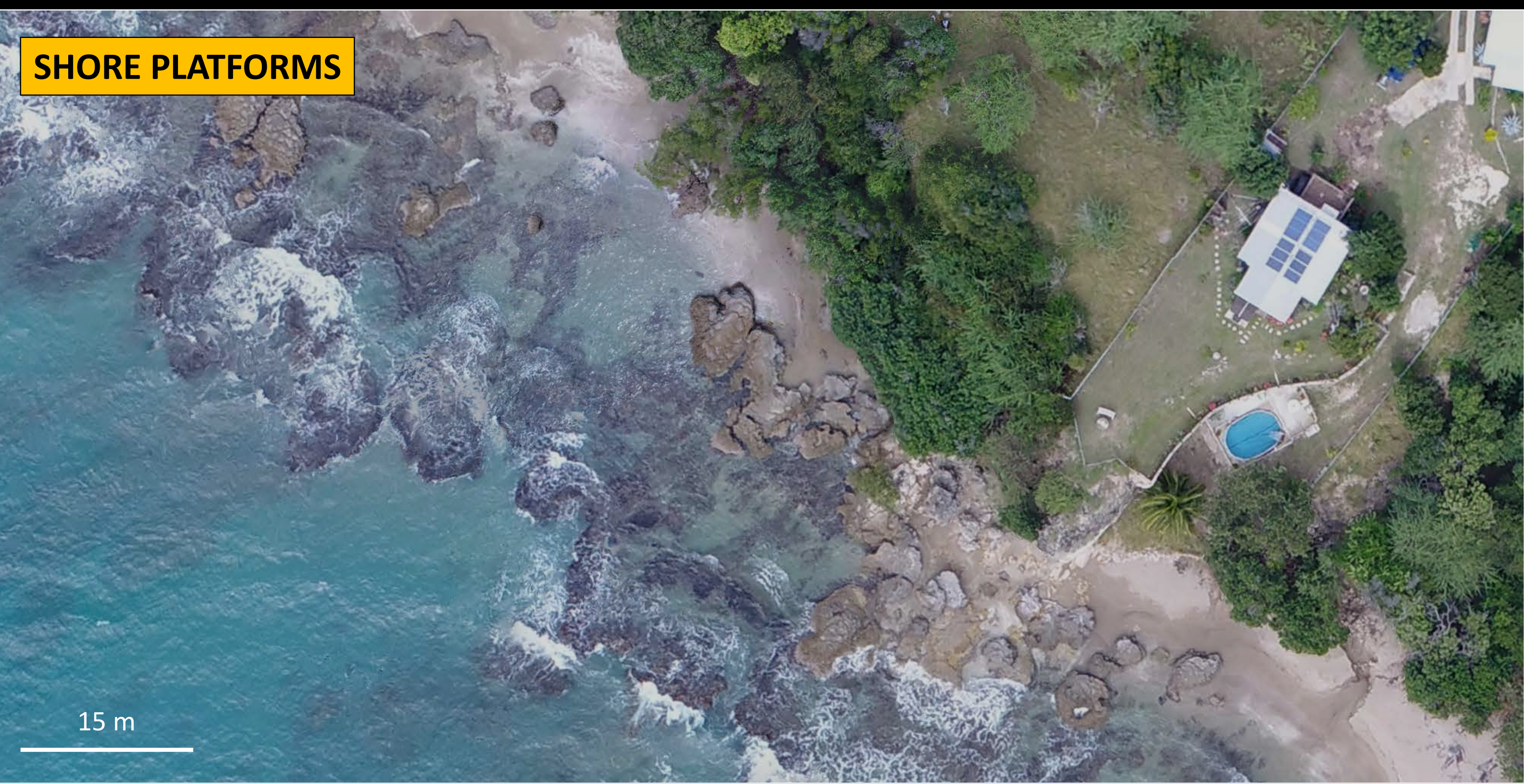




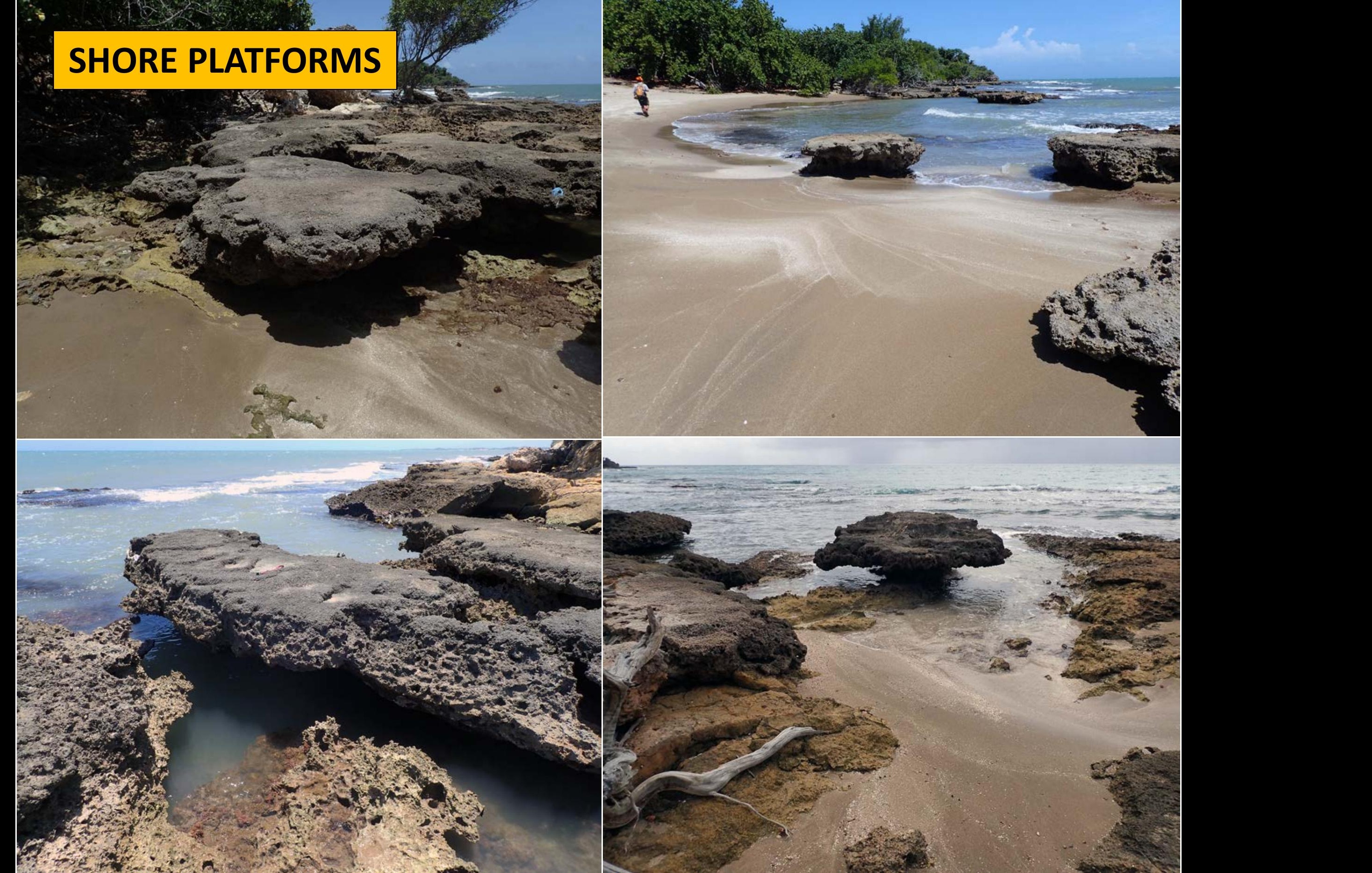




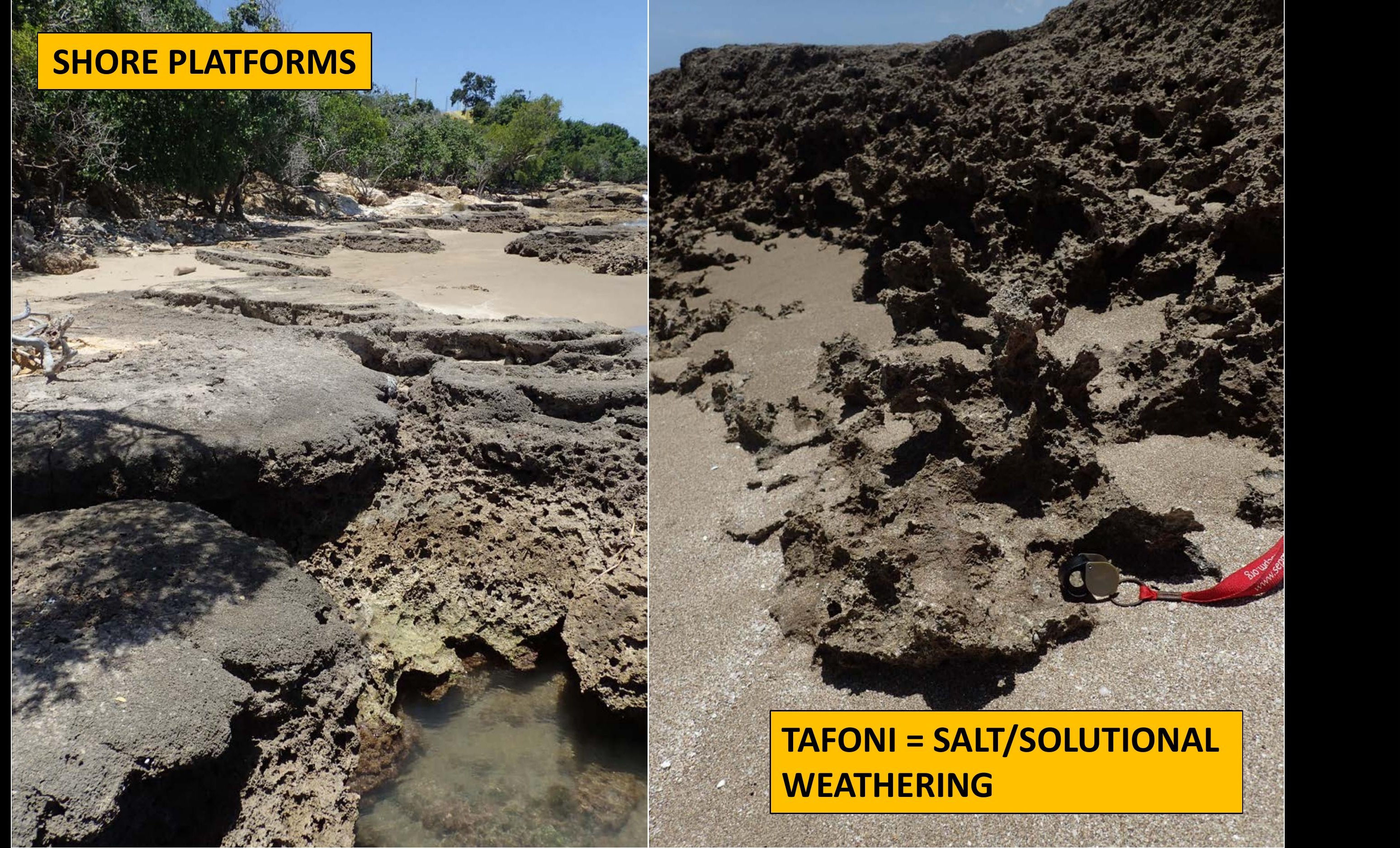




\section{SERPULID ENCRUSTATION ON MIS 5e CORAL}

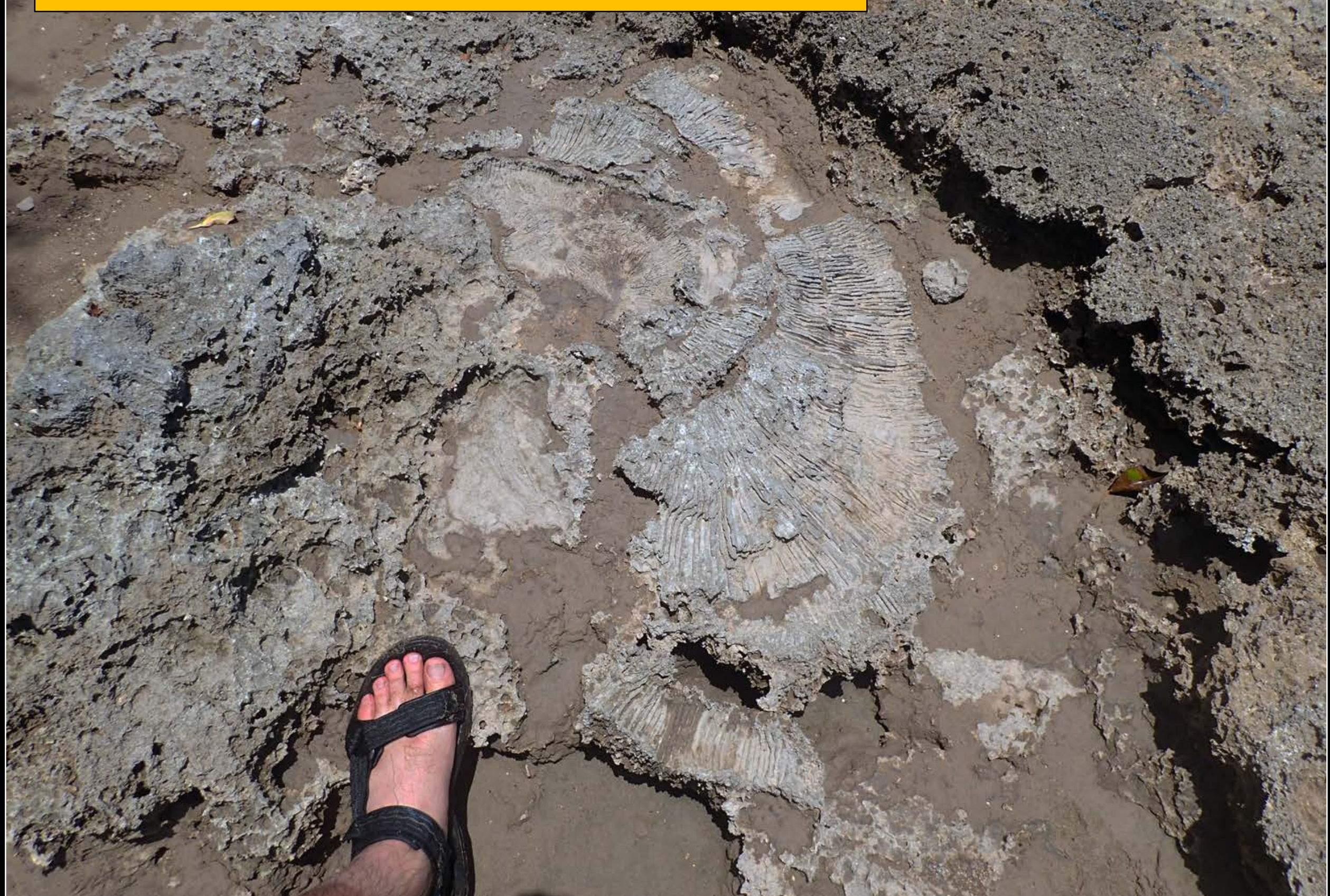




\section{BLACK SPRING POINT}

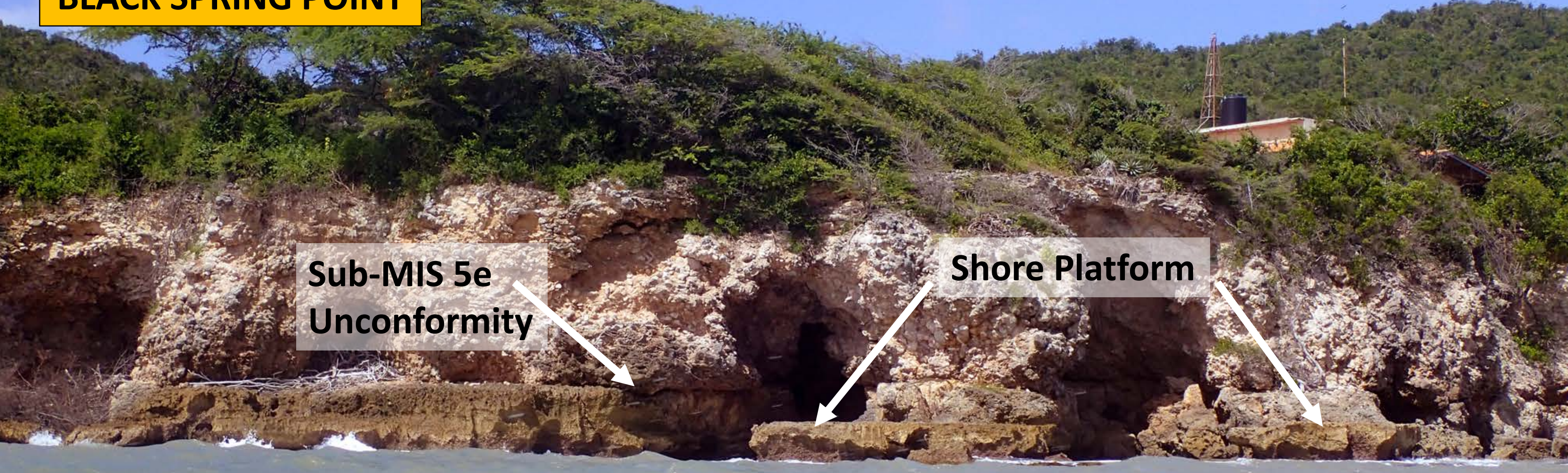




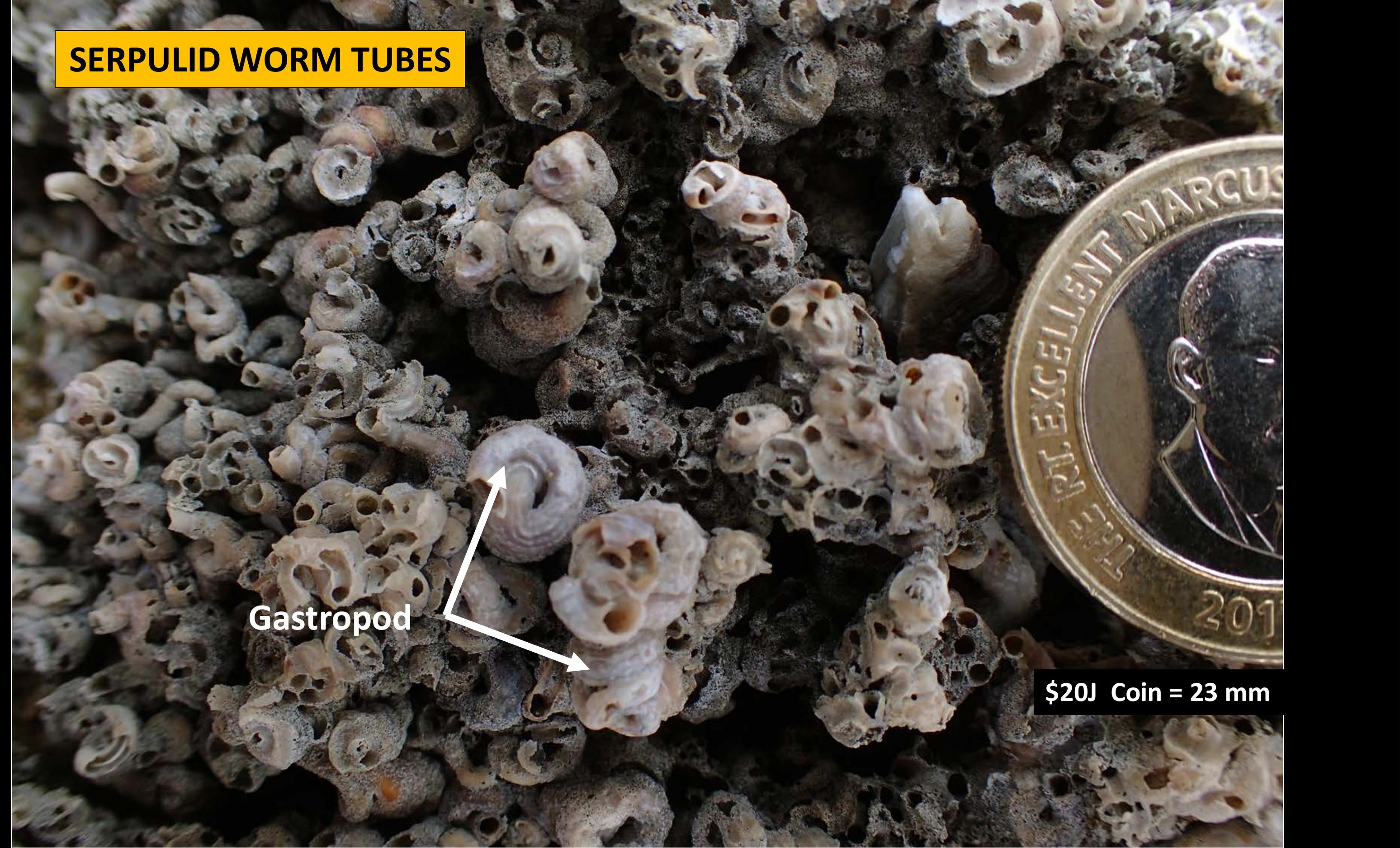




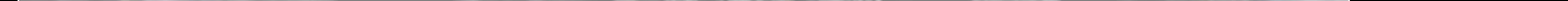


s

CHAMIDAE "JEWEL BOX" BIVALVE SHELLS

(a) $x^{2}$

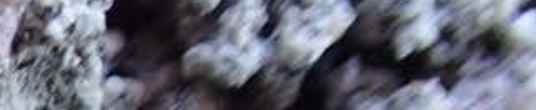

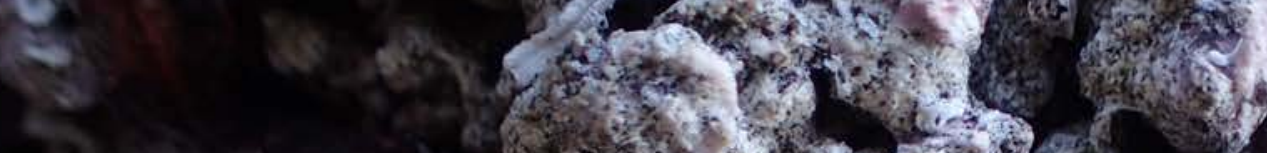

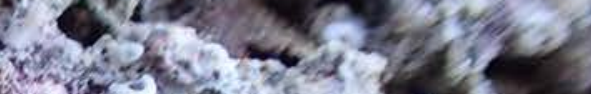

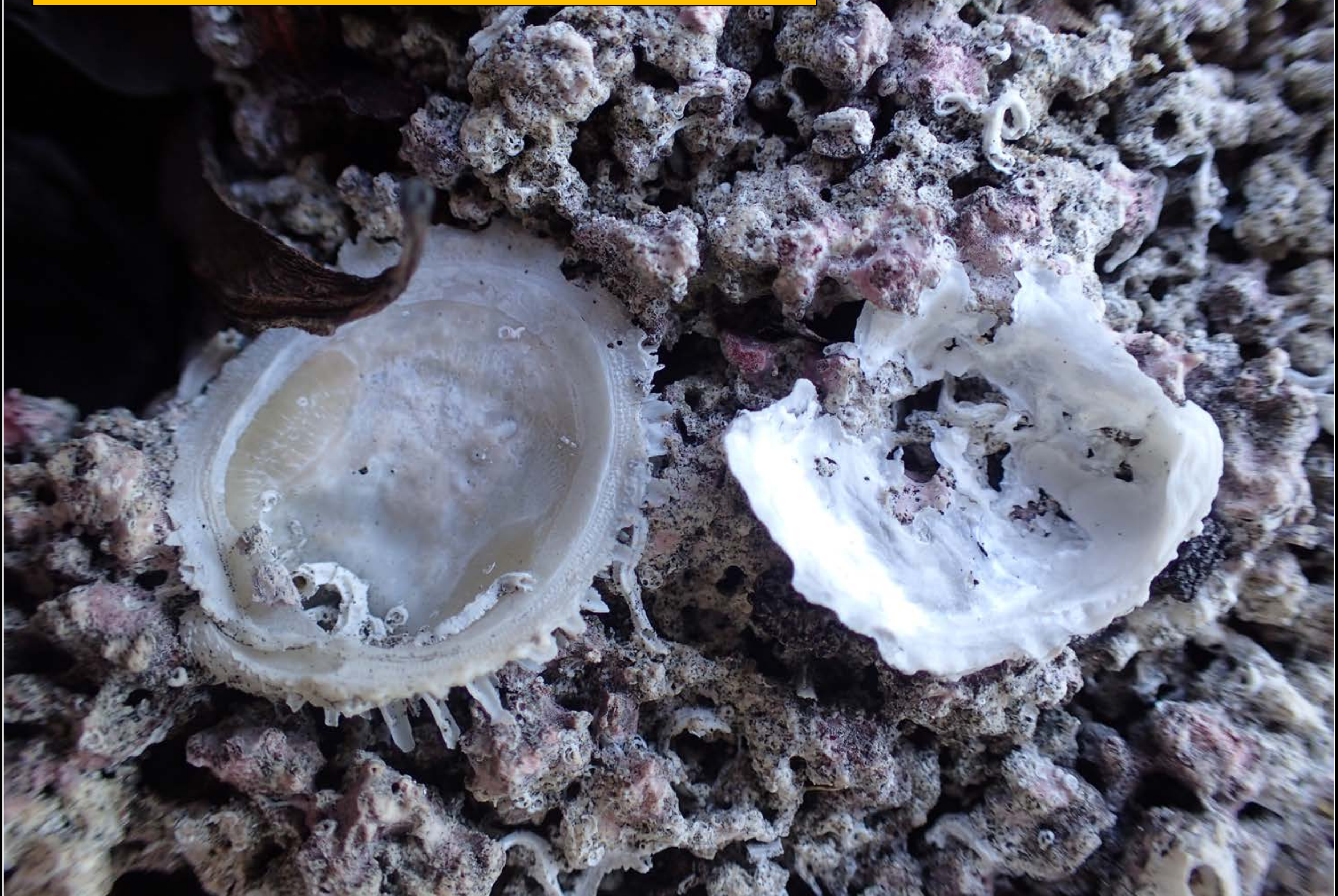




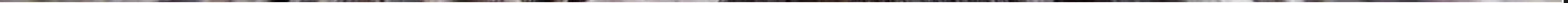




\section{SERPULID WORMS}

- Class Polychaeta

- Suborder Sabellida (fan worms)

- Family Serpulidae

- 490 living species and $\mathbf{7 0}$ genera

- Mid-Triassic to Recent

- Calcareous tubes

- Modern reef-former and encrusting habitats

- Shallow to deep marine

- Tropical to cool temperate climates

\section{AGE DATES AND STABLE ISOTOPE VALUES}

- ${ }^{14} \mathrm{C} 1184-940$ cal BP $(95.4 \% \mathrm{Cl})$
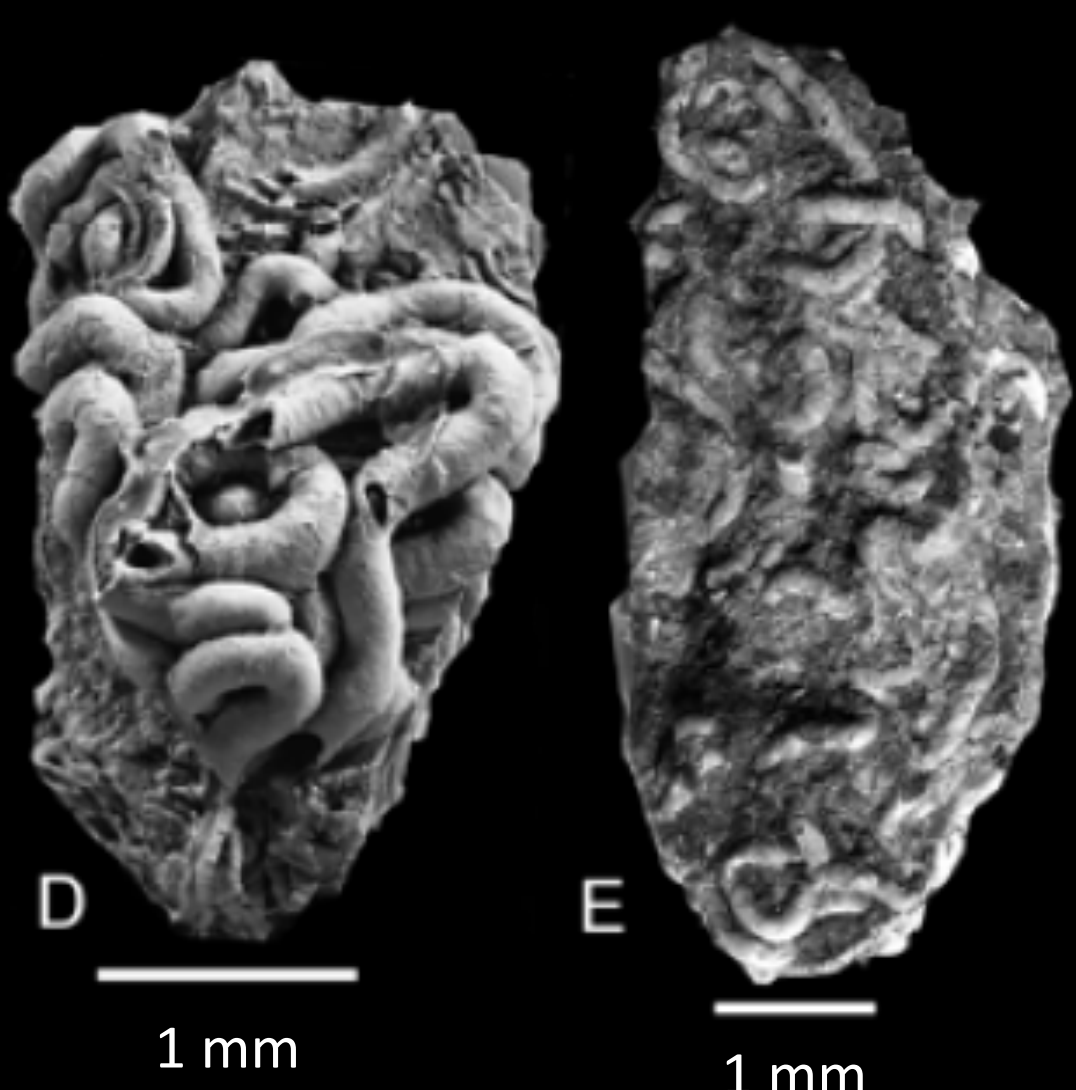

- $952 \pm 122 \mathrm{CE}$

D, "Filogranula" cincta (Goldfuss, 1831)

- $\delta^{13} \mathrm{C}+2.7^{\circ} \%$ vSMOW

E, Metavermilia (Vepreculina) minor Jäger, 1983

- $\delta^{18} \mathrm{O}-1.0$ \% $\%$ Both from Cretaceous, Lower Saxony, Germany Ippolitov et al. (2014) 


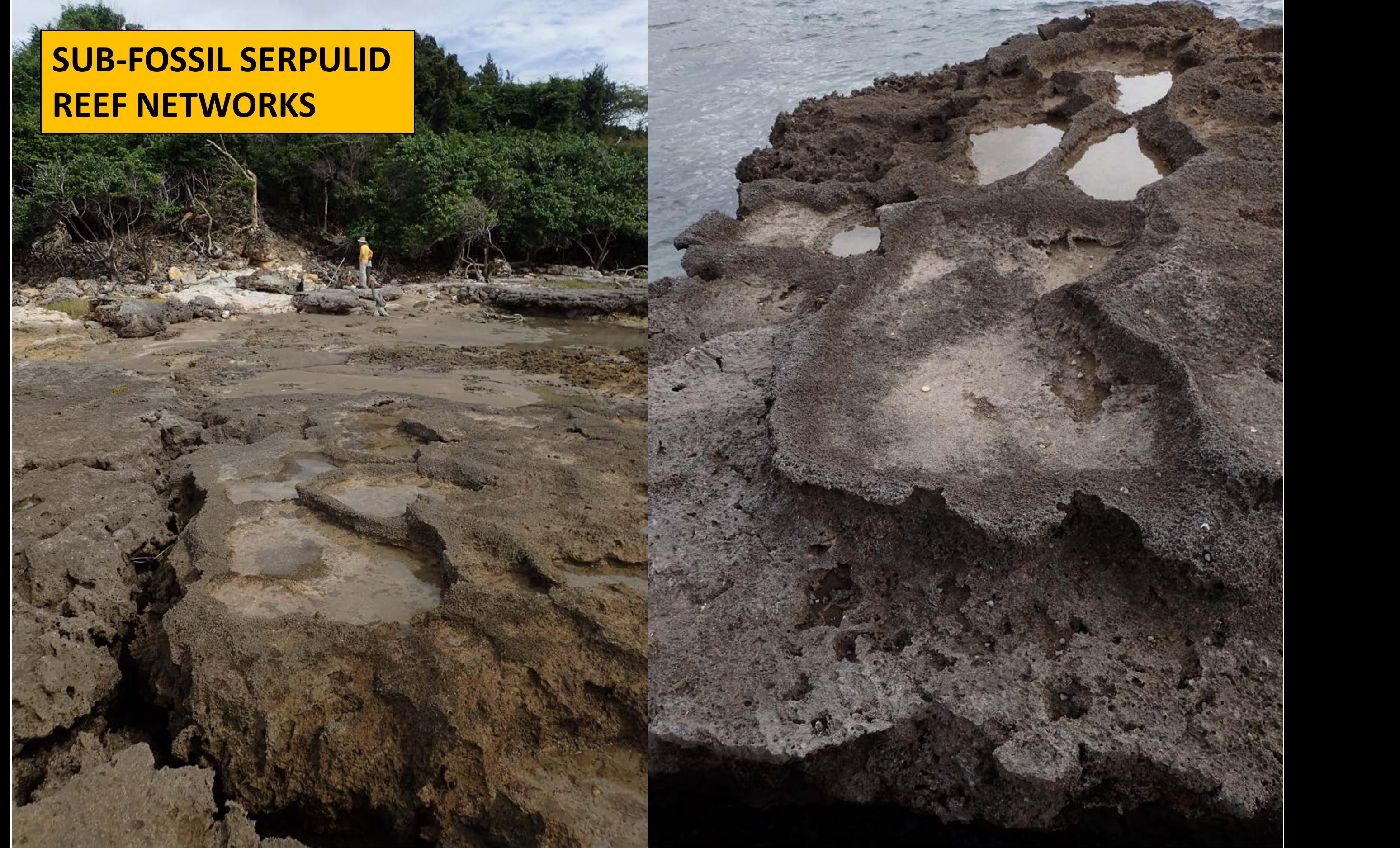




\section{GENERALIZED SHORELINE PROFILE}

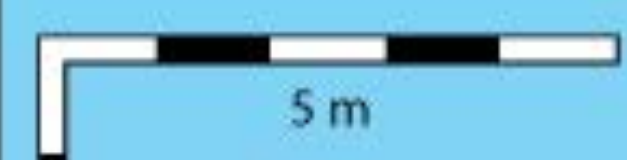

$5 \mathrm{~m}$

Encrusting serpulid worm "reef" raised shore platform $(1184-940 \mathrm{cal}$. BP)

Wave-cut notch (recent) Shore platform (recent)

\section{Eolian sand (Holocene)}

"Old Eolianite" (post-MIS 5e)

Falmouth Formation (MIS 5e)

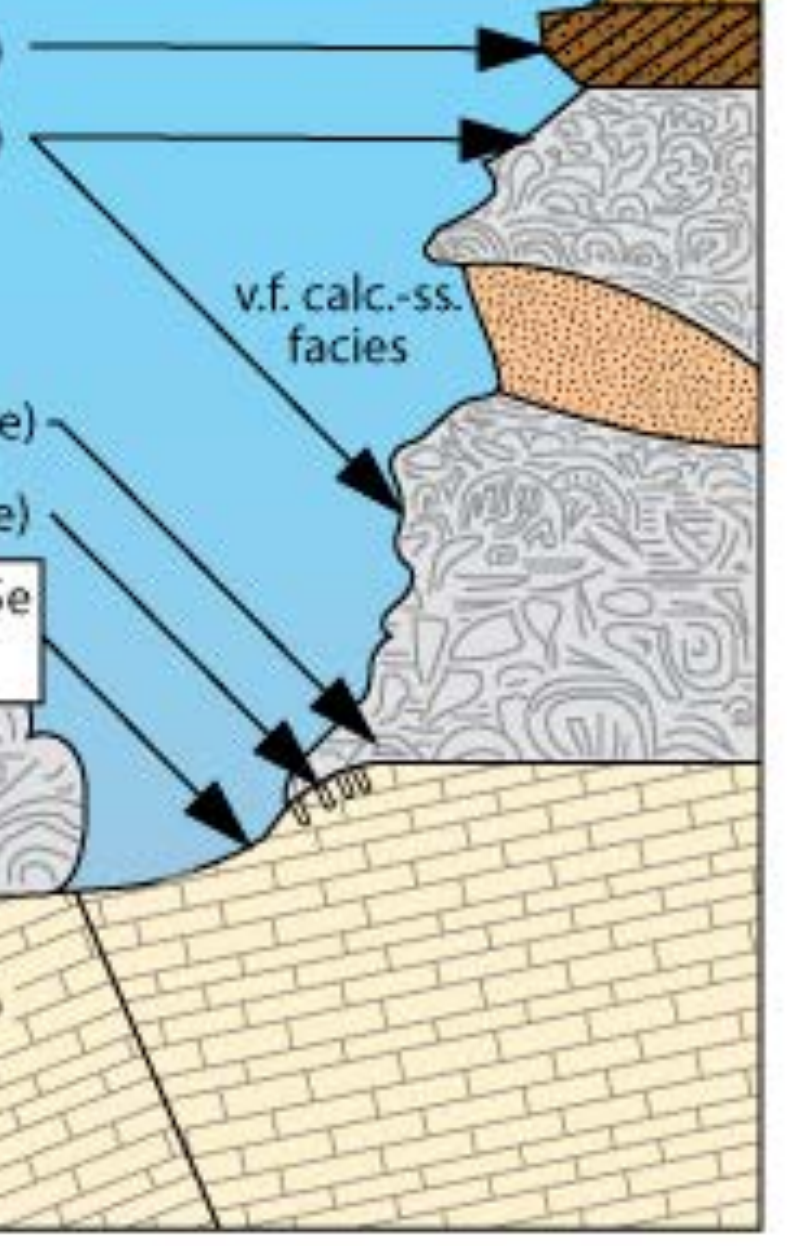

White Limestone Group

(Eocene - Miocene)

Toppled block (MIS Se) - 


\section{STRUCTURAL INTERPRETATION}
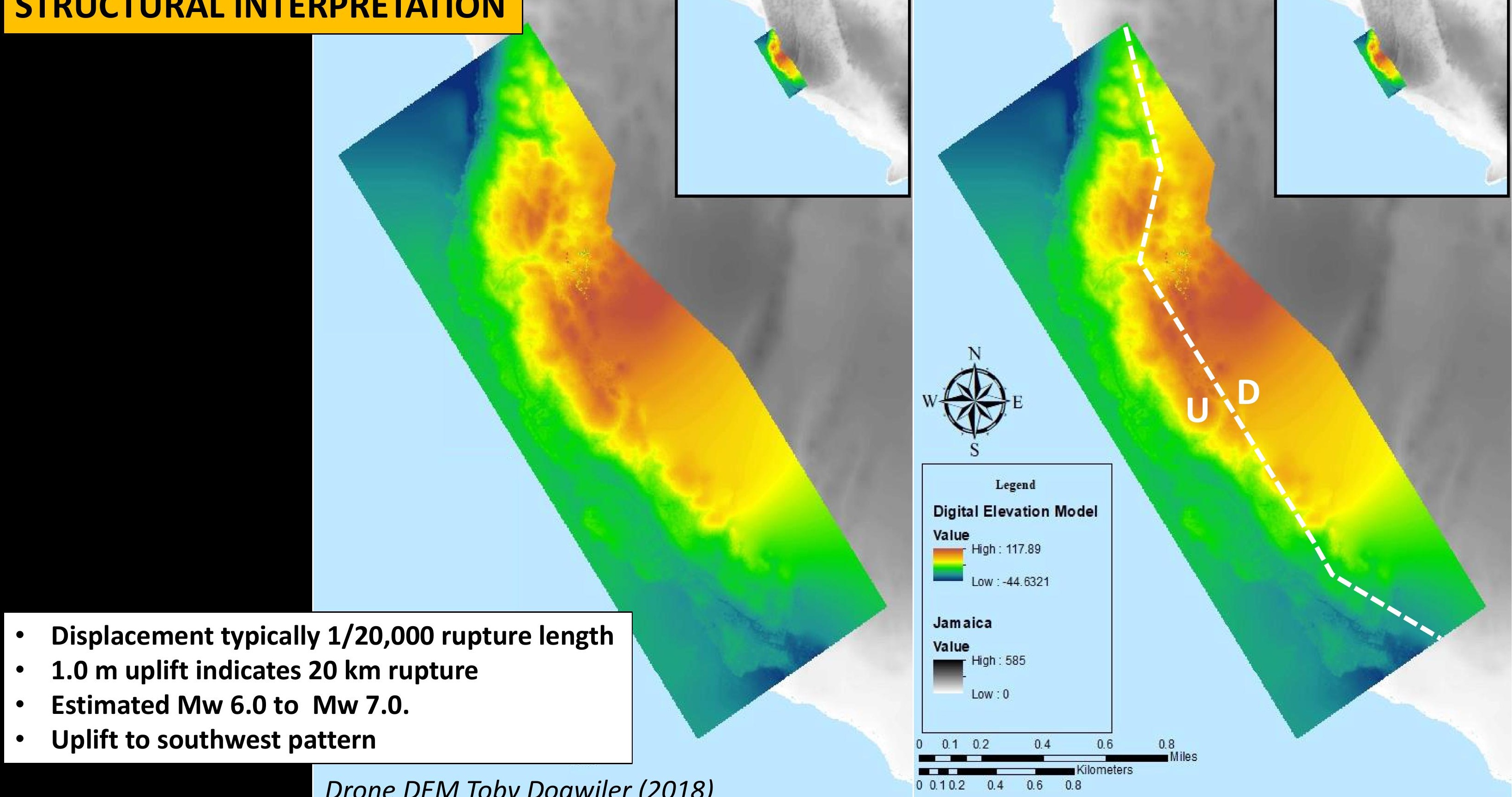
- Jamaica seismically active

- 1.0 m raised shore platforms

- $1184-940$ cal BP ${ }^{14} \mathrm{C}$ age date

- Erosion actively undercutting platforms
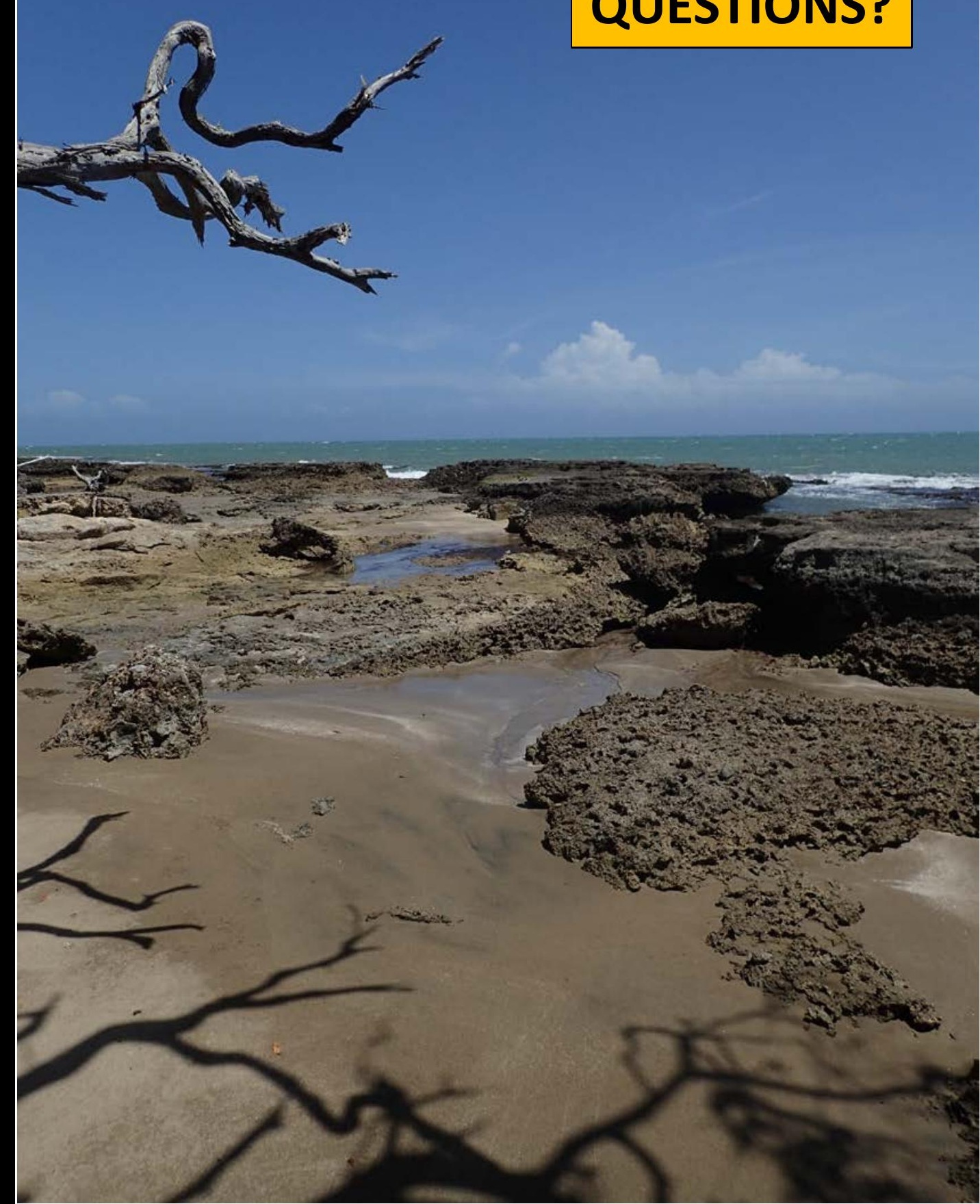